\section{Can the availability of unrestricted financial support improve the quality of care of thalassemics in a center with limited resources? A single center study from India}

\author{
Prantar Chakrabarti, ${ }^{1}$ \\ Vinaykumar Bohara, ${ }^{1}$ Sudeshna Ray, ${ }^{2}$ \\ Siddhartha Sankar Ray, ${ }^{1}$ \\ Uttam Kumar Nath, ${ }^{1}$ Utpal Chaudhuri ${ }^{1}$ \\ 'Institute of Haematology and \\ Transfusion Medicine, Medical College; \\ ${ }^{2}$ All India Institute of Hygiene and Public \\ Health, Kolkata, India
}

\section{Abstract}

Comprehensive management of thalassemia demands a multidisciplinary approach, sufficient financial resources, carefully developed expertise of the care givers, as well as significant compliance on the patients' part. Studies exploring the utility of unrestricted financing within the existing infrastructure, for the management of thalassemia, particularly in the context of a developing country, are scarce. This study aimed to assess the impact of sponsored comprehensive care compared to the routine care of thalassemics provided at Institute of Haematology and Transfusion Medicine, Kolkata, India. Two hundred and twenty patients were selected for the study and distributed in two arms. Regular monthly follow up was done including a Health Related Quality of Life (HRQoL) assessment with SF 36 v2 (validated Bengali version). Patients receiving sponsored comprehensive care showed a significant improvement in the mean hemoglobin levels and decrease in mean ferritin. HRQoL assessment revealed a better score in the physical domain though the mental health domain score was not significantly better at nine months. Unrestricted financial support in the form of comprehensive care has a positive impact on the thalassemia patients in a developing country not only in terms of clinical parameters but also in health related quality of life.

\section{Introduction}

Thalassemias and hemoglobinopathies such as hemoglobins $\mathrm{S}, \mathrm{C}$ and $\mathrm{E}$, are now a global problem with over 300,000 infants with major syndromes being born every year. ${ }^{1}$ It is highly prevalent in those parts of the world, which face the dual problem of illiteracy and poverty. With increased understanding of the natural history of the disease and the development of newer modalities of treatment, the mean survival of thalassemic patients has significantly increased. However, with greater survival have arrived greater responsibilities and challenges, in terms of multidisciplinary clinical management, rehabilitation and social functioning. The comprehensive management of thalassemia demands a multidisciplinary approach, sufficient financial resources, carefully developed expertise of the caregivers, as well as significant compliance on the patients' part.

In the guidelines published by WHO in 1994, Modell ${ }^{2}$ had estimated the cost of only leukodepletion and iron chelation to be $\$ 1720-\$ 6290$ per patient per year. Though oral iron chelators have been subsequently available, the average cost of treating a child with thalassemia is no less than $\$ 2500$ per year in India, which is shared between the state and the family, while per capita income of the state of West Bengal in India is only $\$ 996.54 .^{3}$ Even when blood transfusion is provided free of cost by the government, the incidental expenses (without accounting for iron chelation and leucodepletion) has been estimated to be $\$ 108$ to $\$ 432$ per patient per year at the Institute of Haematology \& Transfusion Medicine (IHTM), Medical College, Kolkata. ${ }^{4}$

The penetration of health insurance in India has been reported to be low. It is estimated that only about $3 \%$ to $5 \%$ of Indians are covered under any form of health insurance. ${ }^{5}$ Hence, in case of a chronic disease like thalassemia, most of the patients do not have any form of insurance coverage and this hampers the management in a comprehensive manner. In terms of infrastructure, the problems are much more in our country, as India has an overall beta thalassemia carrier rate of $4.05 \%{ }^{6}$ The problem is worse in the state of West Bengal where the prevalence of beta thalassemia carriers is estimated to be $10.38 \%$, with another $4.30 \%$ being Hemoglobin E [26(B8)Glu Lys] carriers. $^{7}$ IHTM alone has 1410 registered thalassemics with $72 \%$ of them suffering from Hemoglobin $\mathrm{E}$ beta thalassemia. A percentage of 99.8 of these patients have been categorized by the government as living below the poverty line.

Reeling under the overload of thalassemics, with only $2.8 \%$ of the patients affording any form of iron chelation, we were looking for avenues to at least fund the iron chelation and leucodepletion in addition to the transfusion services being provided. A charity foundation named ONGC Officers' Mahila Samity (OOMS) came forward to sponsor the comprehensive management of around 100 thalassemia patients, as there were not enough funds for all the patients. With the approval of the fund-
Correspondence: Prantar Chakrabarti, Institute of Haematology and Transfusion Medicine, 3rd floor MCH building, Medical College, 88, College Street, Kolkata 700073 India.

Tel: +919433018899 - Fax: +913322123785

E-mail: prantar@ihtmkolkata.in

Key words: thalassemia, financial, chelation, quality of life, SF 36 v2.

Contributions: PC, conceived, designed and implemented the study as a Research Guide, drafted the manuscript; VB, conducted the clinical part of the study, managed all patients and collected all data and analyzed them; SR conducted QOL part of the study, interviewed all patients and collected relevant data and analyzed them; SSR, conducted the clinical study including OPD management and went through the manuscript and analysis; UKN, conducted the clinical study including inpatient and OPD management and went through the manuscript and analysis; UC, helped in the design, implementation and analysis of the study as the Director of the institute.

Conflict of interests: PC, is Advisory Board Member for Novartis Oncology. There are no other conflicts of interest.

Received for publication: 3 September 2012. Revision received: 1 December 2012.

Accepted for publication: 7 December 2012.

This work is licensed under a Creative Commons Attribution 3.0 License (by-nc 3.0).

(C) Copyright P. Chakrabarti et al., 2013

Licensee PAGEPress, Italy

Thalassemia Reports 2013; 3:e2

doi:10.4081/thal.2013.e2

ing agency and the institutional ethical committee, we planned a prospective study to perceive the changes in the quality of life of thalassemics, when unrestricted financial resources are made available to the existing infrastructure.

\section{Materials and Methods}

The study was approved by the Institutional Ethical Committee of Medical College, Kolkata and was conducted in accordance with the International Conference on Harmonization Guidelines on Good Clinical Practice and the principles of the Declaration of Helsinki.

Two Hundred and twenty patients who were at the bottom of the registered list according to their socioeconomic status were selected for the study. After exclusion due to unwillingness for regular follow up $(n=9)$, and expected survival < five years $(\mathrm{n}=3), 208$ patients were randomized into one of the two study arms.

The study started on 1st March 2011 and 
data till December 2011 has been analyzed while the study is continuing. Psychosocial assessment including Health Related Quality of life (HRQoL) evaluation was performed at nine months of follow up using SF 36 v2 (Bengali version) ${ }^{8}$ In one of the arms, intervention in the form of sponsored comprehensive thalassemia care was provided (Jeevan Asha) and the other arm continued receiving thalassemia care which they were taking at their own expense, except for free blood transfusion being provided by the institute (Control).

The clinics for both the study arms were held on different days of the week. The care delivery for the Jeevan Asha group was as follows:

- Special clinics: clinical evaluation of the patients at the time of the visit to the thalassemia clinic with special attention to hematological, cardiac, hepatic, endocrinological status including attainment of puberty (at least once in a month) and implementation of additional non-hematological treatment as and when required e.g. puberty induction with hormonal therapy;

- Blood transfusion: prescheduled packed cell transfusion at the Daycare unit twice a week. An effort was made to maintain pretransfusion hemoglobin of $8 \quad \mathrm{~g} / \mathrm{dL}$. Leukodepletion was performed using bedside leukocyte filters during transfusion with Imugard ${ }^{\circledR}$ III-RC (Terumo Penpol Ltd);

- Nucleic acid testing (NAT) of donated blood: the Procleix Ultrio Assay (Novartis, Emeryville, CA, USA) using TMA technology for simultaneous detection of HIV-1 RNA, HCV RNA, and HBV DNA, was implemented on the semi-automated Procleix eSAS system for routine blood screening using IDNAT format. It may be worthwhile to mention that NAT is not a part of the screening policy of the National Blood Transfusion Service in India;

- Investigations on follow up: serum ferritin (by ELISA method), 2D echo with color doppler study, thyroid function test, FSH assay, LH assay were performed on a regular basis as per institutional guidelines. Magnetic Resonance based iron estimation facilities do not exist in eastern India and hence was not performed.

- Provision of adequate iron chelation: Most of the patients are on deferasirox (ASUNRA, Novartis) at a dose of $40 \mathrm{mg} / \mathrm{kg} /$ day. Those who failed to respond were started on combination therapy. We wanted to use the combination of Deferasirox and Desferioxamine. However due to non-availability of infusion pumps, we were compelled to use a combination of Deferasirox (ASUNRA, Novartis) and Deferiprone (KELFER, Cipla) both of which can be given orally. The combination was used in eight patients in the Jeevan Asha group;

- Reimbursement of individual treatment expenses: all treatment related expenses incurred during hospital admissions, especially the cost of expensive antibiotics, which are not available in the hospital, were reimbursed. Splenectomy and other surgical procedures were fully reimbursed.

In the other arm, none could afford the NAT facility and leucodepletion, while only 20 patients could afford iron chelation at some point of time, but only seven patients were on regular chelation. The battery of minimal investigations could not be afforded by most of the patients.

Over a period of nine months, at least nine visits were recorded for both the study arms. At the end of nine months, an assessment of the psychosocial functioning was done using SF 36 v2 in Bengali.

The SF 36 is a multi-purpose, short-form health survey with only 36 questions. ${ }^{8}$ It yields an 8-scale profile of functional health and wellbeing scores as well as psychometrically-based physical and mental health summary measures and a preference-based health utility index. It is a generic measure, as opposed to one that targets a specific age, disease, or treatment group. SF $36 \mathrm{v} 2$ is a modified version of SF 36, introduced in 1996 to evaluate quali- ty of life in patients with chronic illness. Thirty-five items are used to construct eight scales, which represent physical functioning, social functioning, role limitations due to physical health problems, role limitations due to emotional problems, mental health, vitality, bodily pain and general health perceptions. An additional item measures health transition. Survey response codes are re-coded according to standardised procedures. Higher scores of each subscale (0-100) indicating better HRQoL. The physical and mental components of eight scales were combined into physical component scale (PCS) and mental component scale (MCS) scores. ${ }^{9}$

The sequence of events during the study period is summarized in Figure 1 (CONSORT diagram). The data was analyzed at the end of nine months for the evaluation of both physical and psychosocial profile using SPSS 19.

\section{Results}

All patients enrolled in this study were diagnosed with transfusion dependent thalassemia. Table 1 shows the baseline characteristics of both the treatment arms in the 202 analyzed patients. The two treatment arms

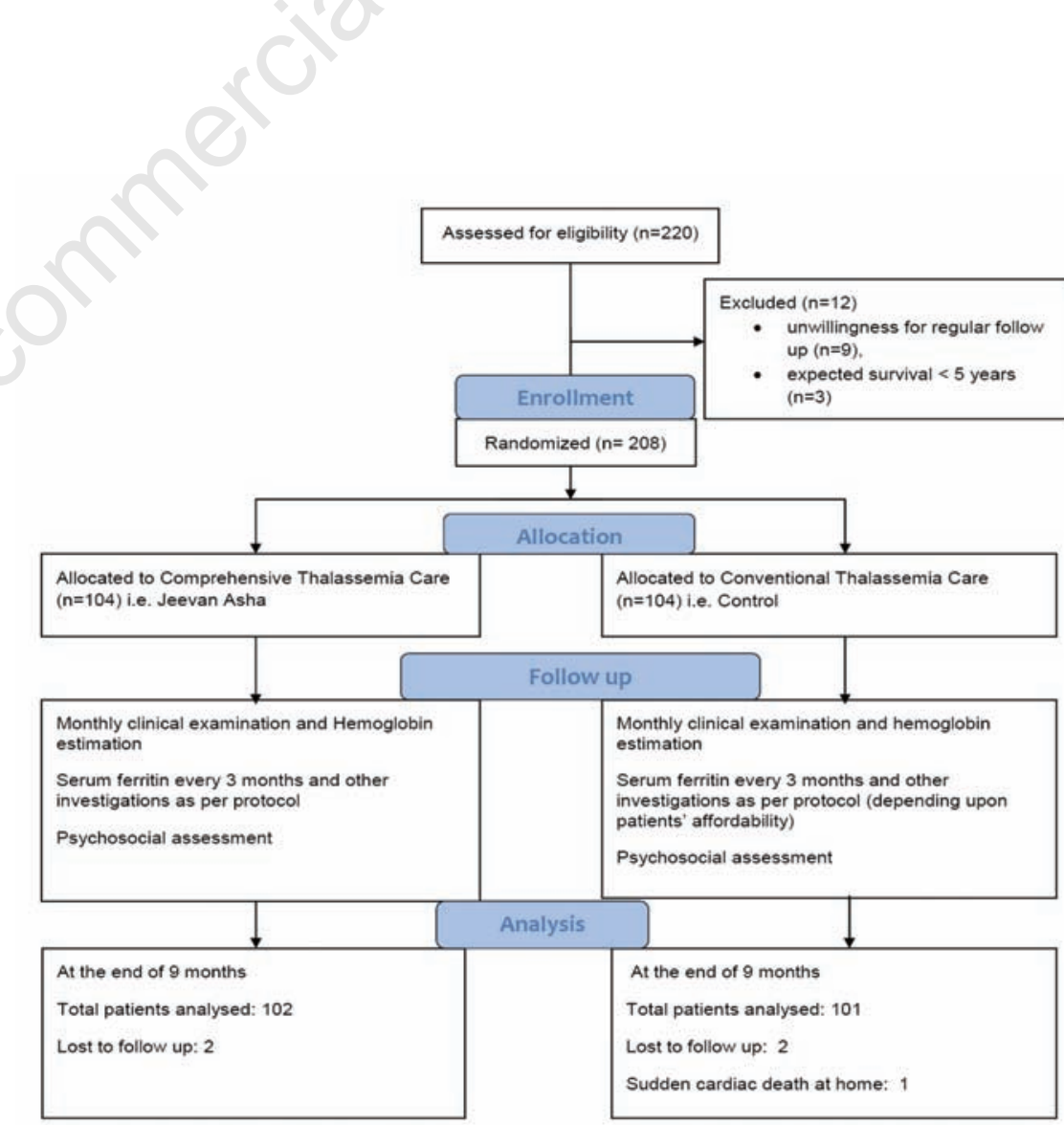

Figure 1. Consort diagram of the study. 
were generally balanced with respect to demographics and baseline disease characteristics. About $70 \%$ of the thalassemics were suffering from Hemoglobin E beta thalassemia but they also had a severe phenotype that required regular blood transfusion.

Table 2 shows mean hemoglobin and mean serum ferritin, respectively in both the study arms at baseline and at nine months. The mean hemoglobin in the Jeevan Asha group was similar to the Control group at baseline, showing an increasing trend from the baseline of $7.12 \mathrm{~g} / \mathrm{dL}$ to $8.18 \mathrm{~g} / \mathrm{dL}$ at nine months of follow up. This was in sharp contrast to the Control group where mean hemoglobin value remained almost static $(6.9 \mathrm{~g} / \mathrm{dL}$ at baseline and $6.42 \mathrm{~g} / \mathrm{dL}$ at nine months).

The mean serum ferritin at the baseline was comparable in both the study arms but the difference started emerging as early as three months (data not shown) and lasted till the final analysis. Mean serum ferritin was lower in the Jeevan Asha group at all the subsequent time points and the difference was shown to be significant (data not shown).

The Jeevan Asha and control groups had 46 and 53 pre-pubertal patients respectively at recruitment. In the Jeevan Asha group, 17.4\% patients attained puberty while being on comprehensive thalassemia care, which was much higher as compared to just $3.8 \%$ patients attaining puberty in the control group during the same period (Table 3 ). The explanation for such favorable effect on growth and induction of puberty in such a short period was probably due to the use of Injection testosterone in males and oestrogen therapy in females, which was decided by the endocrinologist in the team, while the patients in the control group could not even afford hormone level estimation and treatment. None of the patients developed transfusion transmitted infections (HIV, HBV, or $\mathrm{HCV}$ ) during the nine month follow up period in the Jeevan Asha group while two patients were detected to be harboring antibodies against HCV and one patient showed reactivity with HIV ELISA which was subsequently confirmed by Western Blot during the study period, though all the 208 patients had been screened for HIV, HBV, and HCV at enrolment. The incidence of transfusion reactions, minor as well as major was significantly low in Jeevan Asha group as compared to that of the
Control group as shown in Table 3.

While evaluating HRQoL, it was observed that there was a significant difference in mean scores between the patients enrolled for Jeevan Asha and Control groups in all the four domains of the PCS of SF 36 v2 with a better performance by the patients in the Jeevan Asha group (Table 2). While evaluating the components of vitality, social functioning and emotional role of the MCS, a significant difference was detected in the mean scores with the Jeevan Asha group once again performing better. As far as the mental health component of

Table 1. The baseline characteristics of patients in both the treatment arms.

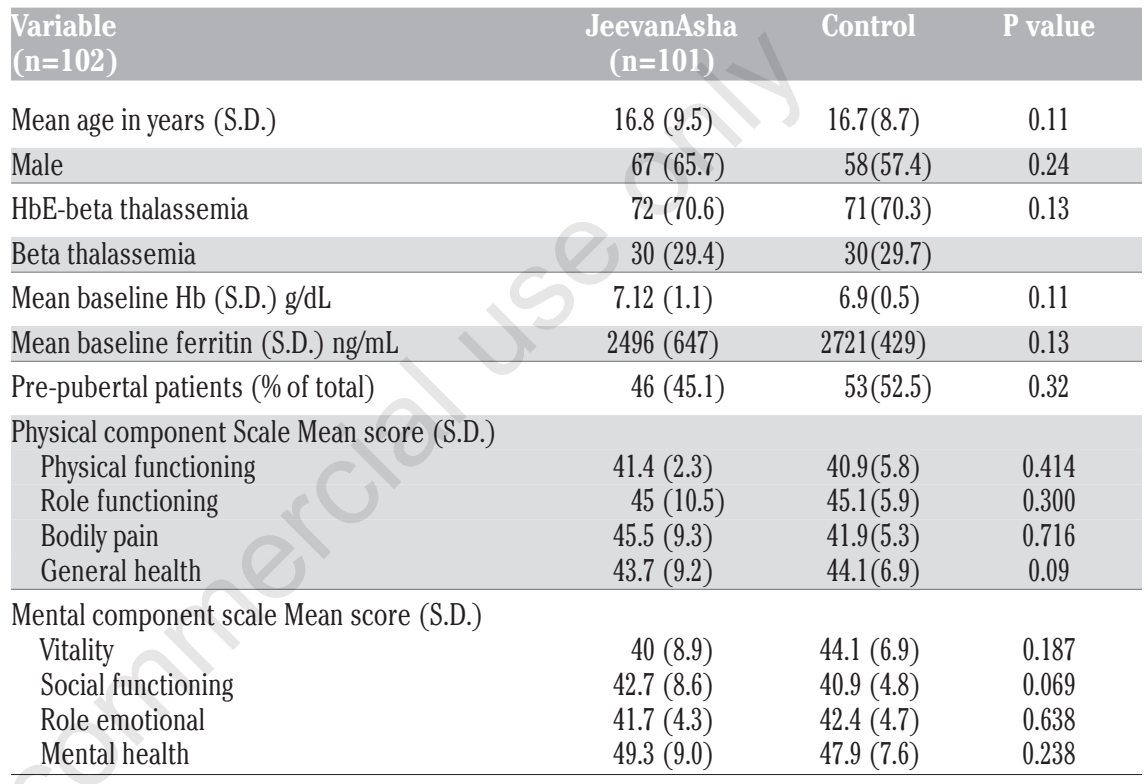

S.D., domains mean score

Table 2. Parameters in both the treatment arms at baseline and after 9 months of care.

\begin{tabular}{|c|c|c|c|c|c|c|}
\hline Study parameters & Baseline & $\begin{array}{l}\text { Jeevanasha } \\
\text { At } 9 \text { months }\end{array}$ & P value (C.I.) & Baseline & $\begin{array}{c}\text { Control } \\
\text { At } 9 \text { months }\end{array}$ & P value (C.I.) \\
\hline Mean Hbgm\% (S.D.) & $7.12(1.10)$ & $8.18(0.70)$ & $<0.001(-1.29$ to -0.84$)$ & $6.90(0.46)$ & $6.42(0.65)$ & $0.08(-0.02$ to 0.33$)$ \\
\hline Mean ferritin ng/mL (S.D.) & $2496(647)$ & $1329(553)$ & $<0.001$ (799.99 to 1078.22) & $2721(428)$ & $2509(901)$ & 0.34 (-193.79 to 572.14$)$ \\
\hline \multicolumn{7}{|l|}{$\begin{array}{l}\text { PCS } \\
\text { S.D. }\end{array}$} \\
\hline Physical functioning & $41.4(2.3)$ & $48.5(10.1)$ & $0.000(-8.80$ to -5.30$)$ & $40.9(5.8)$ & $41.7(6.5)$ & $0.256(-2.1$ to 0.59$)$ \\
\hline Role functioning & $45(10.5)$ & $52.4(10.9)$ & $0.000(-9.0$ to- -5.90$)$ & $45.1(5.9)$ & $45.6(5.7)$ & 0.066 (-3.90 to 0.13$)$ \\
\hline Bodily pain & $45.5(9.3)$ & $49.9(9.3)$ & $0.000(-6.10$ to -2.70$)$ & $41.9(5.3)$ & $45.7(5.8)$ & $0.096(-1.09$ to 0.09$)$ \\
\hline General health & $43.7(9.2)$ & $45.5(9.7)$ & 0.001 (-2.70 to-8.10) & $44.1(6.9)$ & $42.1(5.1)$ & $0.32(-5.97$ to 0.20$)$ \\
\hline \multicolumn{7}{|l|}{$\begin{array}{l}\text { MCS } \\
\text { S.D. }\end{array}$} \\
\hline Vitality & $40(8.9)$ & $49(9.8)$ & $0.000(-4.80$ to- 1.90$)$ & $44.1(6.9)$ & $42.9(7.5)$ & $0.083(-1.61$ to 2.56$)$ \\
\hline Social functioning & $42.7(8.6)$ & $47.9(10.5)$ & $0.000(-6.80$ to -3.60$)$ & $40.9(4.8)$ & $41.1(8.6)$ & $0.9(-2.09$ to 1.69$)$ \\
\hline Role emotional & $41.7(4.3)$ & $48.0(9.2)$ & $0.000(-7.78$ to -4.82$)$ & $42.4(4.7)$ & $42.7(4.9)$ & 0.650 (-1.61 to 1.01$)$ \\
\hline Mental health & $49.3(9.0)$ & $49.8(8.6)$ & 0.059 (-1.01 to 0.08) & $47.9(7.6)$ & $48.1(7.1)$ & $0.326(-6.00$ to 0.20$)$ \\
\hline
\end{tabular}

PCS, physical component scale; MCS, mental component scale; S.D., domains mean score. 
MCS was concerned, it was better in the Jeevan Asha group compared to the Control but it did not achieve statistical significance (Table 2).

\section{Discussion}

Quality health care is a complex phenomenon. The factors contributing to quality in health care are as varied as the strategies needed to achieve this elusive goal. ${ }^{10}$ Limited funding for the health sector is at the root of many country capacity challenges. World Health Organization has earmarked health financing as the key to improving health and reducing health inequities. The objective of this study was to assess the impact of unrestricted financial support in improving the physical and psychosocial health of thalassemia patients. In our search for similar studies, we failed to identify any such study especially in a country like India probably due to the fact that unrestricted financial help is rarely available to patients who themselves bear the entire expenses of the treatment. Lack of adequate health care spending inevitably leads to under-development of infrastructure and inequitable distribution of services. Safe and optimal blood transfusion and adequate iron chelation are the pillars of basic thalassemia care, which becomes a major challenge in resource-limited countries. The difficulties faced in keeping baseline hemoglobin near the recommended range are many-fold in such countries and these include shortage of day care facilities for transfusion, concentration of health care services in urban areas and shortage of blood banking services due to technical and financial limitations. We observed a higher pre-transfusion hemoglobin levels in the Jeevan Asha group during the follow up. This was probably due to better compliance, motivation on the part of the patients and their families who were sure of the availability of the facilities including NAT screening and bedside leukodepletion.

Over the past four decades, the survival of thalassemia patients has almost doubled and adequate iron chelation has been the most important contributor. However, around $87 \%$ of children born with thalassemia major die untreated, while at least $60 \%$ of those who start on regular transfusions have no access to iron chelation therapy. ${ }^{11}$ In our country, probably less than $10 \%$ of thalassemia patients can afford to take regular chelation as per the recommended guidelines. In fact, the death of a patient which occurred in the control group was probably consequent to iron overload. The death occurred at home and was informed by the patient's relatives. As per history described, it was assumed to be a sudden car-

Table 3. Table showing attainment of puberty, incidence of transfusion transmitted infections, and incidence of transfusion reactions in both study arms.

\begin{tabular}{|c|c|c|c|}
\hline $\begin{array}{l}\text { Parameter } \\
(n=102)\end{array}$ & $\begin{array}{c}\text { JeevanAsha } \\
(\mathrm{n}=101)\end{array}$ & $\begin{array}{l}\text { Control } \\
\text { ( } 95 \% \text { C.I.) }\end{array}$ & P value \\
\hline Puberty attained & $8 / 46(17.4 \%)$ & $2 / 53(3.8 \%)$ & $0.04^{*}(1.07$ to 26.7$)$ \\
\hline $\begin{array}{l}\text { Incidence of TTI during study period } \\
\text { HIV } \\
\text { Hepatitis B virus } \\
\text { Hepatitis C virus }\end{array}$ & $\begin{array}{l}0 \\
0 \\
0\end{array}$ & $\begin{array}{c}1(0.9) \\
0 \\
2(1.9)\end{array}$ & - \\
\hline $\begin{array}{l}\text { Incidence of transfusion reactions during study period } \\
\text { Non hemolytic febrile transfusion reaction } \\
\text { Hemolytic transfusion reaction }\end{array}$ & $\begin{array}{l}7(6.9) \\
1(0.9)\end{array}$ & $\begin{array}{c}40(39.6) \\
1(0.9)\end{array}$ & $\begin{array}{c}<0.001^{*} \\
(-0.38 \text { to }-0.17)\end{array}$ \\
\hline
\end{tabular}

*Denotes statistically significant. TTIs, transfusion transmitted infections.

diac death. The provision of oral iron chelation for all the eligible patients in the Jeevan Asha group caused a drop in mean serum ferritin from $2496 \mathrm{ng} / \mathrm{dL}$ to $1329 \mathrm{ng} / \mathrm{dL}$ at the end of nine months. On the other hand, 20 (19.8\%) in the control group were affording iron chelation at the time of entry in study. The number further decreased to $16(15.8 \%), 10(9.9 \%)$ and 6 (5.9\%) at the end of three, six and nine months respectively in spite of regular psychosocial counseling. This drop out was solely due to financial reasons. Even in patients who were on chelation, detailed interrogation revealed that most were not taking the dose prescribed and there was lack of continuity due to non-availability of funds. Thus, there was hardly any difference in the mean ferritin level at baseline $(2721 \mathrm{ng} / \mathrm{mL})$ and at the end of nine months $(2509 \mathrm{ng} / \mathrm{mL})$ in the control group. Even when funds were available in the Jeevan Asha group, non-responders could not be started on combination therapy of deferasirox with desferrioxamine due to nonavailability of fixed-dose infusion pumps in the country. As a consequence of inadequate iron chelation, endocrine abnormalities like growth retardation and delayed puberty are well known in Indian thalassemics. ${ }^{12}$ The situation was similar in our study at enrolment. What was striking was that, in the Jeevan Asha group, approximately $17.4 \%$ patients attained puberty with hormonal induction treatment within nine months, while only $3.8 \%$ of total pre-pubertal patients attained puberty in the control group during the same period where none of the patients could afford it. Though the incidence of transfusion transmitted infections like HIV, HBV and HCV infections in multi-transfused thalassemics has decreased to a large extent with the introduction of serological tests, even after implementing the more sensitive, newest generation of serological tests, a considerable residual risk of transfusion transmission of these viruses remains. Although the more sensitive serological tests have shortened the pre-seroconversion window period, they still are not able to identify a number of newly infected donors. ${ }^{13}$ These undetected window period infections are responsible for most of the transfusion transmission of these viruses. NAT is currently used in conjunction with serological tests in the developed countries. ${ }^{14}$ Although NAT screening cannot completely eliminate the risk of transfusion transmitted infection, it has reduced the risk for HIV-1 and HCV where it has been implemented. ${ }^{15}$ We introduced NAT for HIV, HBV and HCV on the donated blood meant for transfusion to the Jeevan Asha patients through the fund provided by 00MS as a pilot project. The annual yield for positive NAT was 9 for HCV out of 1394 samples tested, which had been missed by ELISA screening at the blood bank. As a result, the incidence of new seroconversion amongst the Jeevan Asha patients was zero for all three infections while 1 and 2 patients in the Control group respectively seroconverted for HIV and HCV (these patients were receiving ELISA screened blood from the blood bank). The incidence of transfusion reactions was also low in Jeevan Asha as compared to the Control group, which may be attributed to the use of bedside leukodepletion. Thalassaemia being a chronic disease has a negative impact on perceived physical functioning, social functioning, bodily pain and general health when compared to the normal population. It has been observed that the overall HRQoL in individuals with thalassemia is largely influenced by the impact of the long term treatment like frequent hospital visits for transfusion, nightly subcutaneous infusions, physical appearance, delayed or absent sexual development, complications of the disease and therapeutic interventions and uncertainties about the future. However, morbidity and mortality related to thalassemia have been reduced significantly with modern medical treatment, and HRQoL should now be considered as an important index of effective health care. ${ }^{16,17} \mathrm{An}$ assessment of HRQoL differs from other forms of medical assessment in that it focuses on the individuals' own views of their well-being and assesses other aspects of life, giving a more holistic view of well-being.

There is very little published work on evaluation of HRQoL in Indian thalassemics either as a part of an international study ${ }^{18}$ or as a sin- 
gle center evaluation ${ }^{19,20}$ especially from eastern India. ${ }^{21}$

In our study, the overall SF 36 v2 scores obtained were somewhat lower in both the physical component scale as well as the mental component scale for Jeevan Asha and Control patients as compared to the general population. This corroborates with similar findings by Shaligram et al. ${ }^{19}$ who reported that the thalassemic children face a lot of social problems related to career, finding partners, establishing a family (due to infertility), and waning social support. However, the Jeevan Asha patients had demonstrated a significantly better score as compared to the Control patients in all the four domains of the PCS, and three domains of the MCS namely vitality, role emotional and social functioning of SF $36 \mathrm{v} 2$. In a study conducted on Thai children, ${ }^{22}$ it was found that pre-transfusion hemoglobin level, frequency of blood transfusion and disease severity were significant predictors of HRQoL of thalassemic children in Thailand. In our study, the Jeevan Asha Patients had a better score as compared to the Control group because of regular pre-planned blood transfusion. The worse score in the Control patients could be attributed to their irregular blood transfusion and lack of affordability of iron chelation.

A recent study conducted on Thai children, ${ }^{23}$ showed that HRQoL had no significant differences by age, gender, type of thalassemia or serum ferritin level. A study from $\mathrm{UK},{ }^{24}$ has shown that iron chelation therapy negatively impacts HRQoL, as compliance with iron chelation is poor, with their patients not achieving target serum ferritin thresholds despite chronic treatment for iron overload. However, in our study, the patients in Jeevan Asha group were not only compliant, when the chelators were available free of cost, but also achieved a significant fall in ferritin level which was also reflected in a better HRQoL score.

In terms of mental health domain of mental component scale of SF 36 v2, Jeevan Asha patients seemed to be faring no better off than the Control patients. This might be due to the financial insecurity and a premonition of stoppage of financial assistance once the project is over, which probably played a detrimental role on their mental health. This finding is supported by a study conducted in Thailand ${ }^{25}$ where the HRQoL in children with thalassemia was not only determined by disease severity and treatment but also by family financial impacts for caring of children. Changes in the mental health of the patients and their family caregivers may require considerable time for a positive impact and mere nine months of financial support may not be sufficient to reflect any change. Appreciating the fact that, there could be other dimensions of management beyond financing and infrastructure which could provide a comprehensive care for the thalassemic patients, we have now come up with the idea of drama therapy, art and elocution competitions to contribute positively to their mental health domain and we hope to report our findings in future.

\section{References}

1. Angastiniotis M, Modell B. Global epidemiology of hemoglobin disorders. Ann N Y Acad Sci 1998;850:251-69.

2. Modell B. Guidelines for the control of haemoglobin disorders. In: VI Annual Meeting of the WHO Working Group on Haemoglobinopathies. Geneva: World Health Organization; 1989.

3. Press Information Bureau - Government of India. Statement: per capita net state domestic product at current price. Available from: http://pib.nic.in/archieve/ others/2012/mar/d2012032902.pdf

4. Mallik S, Chatterjee C, Mandal PK, et al. Expenditure to treat thalassemia - An experience at a tertiary care hospital in India. Iranian J Public Health 2010;39:7884.

5. Rao KS. Health insurance in India. In: Ministry of Health and Family Welfare. Financing and delivery of health care services in India. New Delhi: Governemnt of India; 2005. pp. 275-295. Available from: http:/www.who.int/macrohealth/action/Ba ckground\%20Papers\%20report.pdf

6. Madan N, Sharma S, Sood SK, et al. Frequency of beta-thalassemia trait and other hemoglobinopathies in northern and western India. Indian $\mathrm{J}$ Hum Genet 2010;16:16-25.

7. Dolai TK, Dutta S, Bhattacharyya M, Ghosh MK. Prevalence of hemoglobinopathies in rural Bengal, India. Hemoglobin 2012;36:57-63.

8. Ware JE Jr, Gandek B. Overview of the SF36 Health Survey and the International Quality of Life Assessment (IQOLA) Project. J Clin Epidemiol 1998:51:903-12.

9. Sobota A, Yamashita R, Xu Y, et al. Quality of life in thalassemia: a comparison of SF36 results from the thalassemia longitudinal cohort to reported literature and the US norms. Am J Hematol 2011;86:92-5.

10. Huston CJ. Quality health care in an era of limited resources. Challenges and opportunities. J Nurs Care Qual 2003;18:295301.

11. Modell B, Darlison M. Global epidemiology of haemoglobin disorders and derived service indicators. Bull World Health Organ 2008;86:480-7.

12. Merchant RH, Shirodkar A, Ahmed J.
Evaluation of growth, puberty and endocrine dysfunctions in relation to iron overload in multi transfused Indian thalassemia patients. Indian J Pediatr 2011;78:679-83.

13. Busch MP, Glynn SA, Stramer SL, et al. A new strategy for estimating risks of transfusion-transmitted viral infections based on rates of detection of recently infected donors. Transfusion 2005;45:254-64.

14. Roth WK, Busch MP, Schuller A, et al. International survey on NAT testing of blood donations: expanding implementation and yield from 1999 to 2009. Vox Sang 2012;102:82-90.

15. Stramer Sl, Glynn SA, Kleinman SH, et al. Detection of HIV-1 and HCV infections among antibody-negative blood donors by nucleic acid-amplification testing. N Engl J Med 2004;351:760-8.

16. Pakbaz Z, Treadwell M, Yamashita R, et al. Quality of life in patients with thalassemia intermedia compared to thalassemia major. Ann N Y Acad Sci 2005;1054:457-61.

17. Telfer P, Constantindou G, Anderou P, et al. Quality of life in thalassemia. Ann N Y Acad Sci 2005;1054:273-82.

18. Caro JJ., et al. Impact of thalassemia major on patients and their families. Acta Haematol 2002;107:150-7.

19. Shaligram D, Girimaji SC, Chaturvedi SK. Psychological problems and quality of life in children with thalassemia. Indian $\mathrm{J}$ Pediatr 2007;74:727-30.

20. Shaligram D, Girimaji SC, Chaturvedi SK. Quality of life issues in caregivers of youngsters with thalassemia. Indian $\mathrm{J}$ Pediatr 2007;74:275-8.

21. Roy T, Chatterjee SC. The experiences of adolescents with thalassemia in West Bengal, India. Qual Health Res 2007;1:8593.

22. Thavorncharoensap M, Torcharus N, Nuchprayoon I, et al. Factors affecting health-related quality of life in Thai children with thalassemia. BMC Blood Disord 2010;10:1.

23. Torcharus K, Pankaew T. Health-related quality of life in Thai thalassemic children treated with iron chelation. Southeast Asian J Trop Med Public Health 2011;42: 951-9.

24. Payne KA, et al. Iron chelation therapy: clinical effectiveness, economic burden and quality of life in patients with iron overload. Adv Ther 2008;25:725-42.

25. Surapolchai P, Satayasai W, Sinlapamongkolkul P, Udomsubpayakul U. Biopsychosocial predictors of health-related quality of life in children with thalassemia in Thammasat University Hospital. J Med Assoc Thai 2010;93 Suppl 7:S65-S75. 